\title{
Anterograde Amnesia for Pavlovian Fear Conditioning and the Role of One-Trial Overshadowing: Effects of Preconditioning Exposures to Morphine in the Rat
}

\author{
Gavan P. McNally and R. Frederick Westbrook \\ University of New South Wales
}

\begin{abstract}
Four experiments studied anterograde deficits in Pavlovian fear conditioning following prolonged exposure to the $\mu$-opioid receptor agonist morphine. Injections of morphine produced temporally graded anterograde amnesia characterized by deficits in contextual and conditioned-stimulus (CS) conditioning 1 or 7 days and selective impairment in CS conditioning 21 days after last injection. This anterograde deficit in conditioning did not recover across a retention interval, was absent when rats were tested immediately after conditioning, and required the presence of an auditory CS. These results suggest that anterograde deficits in Pavlovian fear conditioning emerged from differences in susceptibility to 1-trial overshadowing of context by CS.
\end{abstract}

Rats learn to fear an auditory conditioned stimulus (CS) whose presentations signal impending aversive stimulation (e.g., a footshock unconditioned stimulus [US]). They also learn to fear the context where these CS-US pairings occur. Although learned at the same time, these fears can be dissociated. For example, rats subjected to dorsal hippocampal lesions shortly (e.g., 1 day) after fear conditioning exhibit fear reactions (freezing) when subsequently tested with the CS but reduced freezing when reexposed to the shocked context (e.g., Anagnostaras, Maren, \& Fanselow, 1999; Kim \& Fanselow, 1992; Maren, Aharonov, \& Fanselow, 1997). However, rats lesioned some time (e.g., 28-100 days) after conditioning exhibit intact freezing reactions when tested with the CS or reexposed to the shocked context. Similar, context-specific, temporally graded retrograde deficits have been reported following a variety of manipulations imposed on rats in the hours following fear conditioning. Such manipulations include social isolation (Rudy, 1996), immune activation by injection of the bacterial endotoxin lipopolysaccharide (Pugh et al., 1998), and injection of an opioid receptor agonist such as morphine (Rudy, Kuwagama, \& Pugh, 1999).

We have recently shown that prolonged exposure to the $\mu$-opioid receptor (MOR) agonist morphine in the days to weeks following fear conditioning produces temporally bound retrograde amnesia for contextual fear conditioning (McNally \& Westbrook, 2003). In those experiments, rats were subject to pairings of an auditory CS with footshock in a distinctive context. Rats were then injected daily for 2 weeks with morphine. These injections com-

Gavan P. McNally and R. Frederick Westbrook, School of Psychology, University of New South Wales, Sydney, New South Wales, Australia.

These experiments were supported by National Health and Medical Research Grant 209577 and Australian Research Council Grants A00103609 and A1000715. We thank Marika Gorissen for assistance with these experiments.

Correspondence concerning this article should be addressed to Gavan P. McNally, School of Psychology, University of New South Wales, Sydney, New South Wales 2052, Australia. E-mail: g.mcnally@unsw.edu.au menced at varying intervals following conditioning. The results showed that exposures to morphine produced a temporally graded and context-specific retrograde deficit in fear conditioning. This retrograde deficit was accompanied by a failure of contextual discrimination such that the fear displayed by morphine-treated animals generalized completely to a second, novel context. Moreover, brief preexposures protected context fear conditioning and context discrimination against the amnestic effects of postconditioning exposures to morphine.

These retrograde impairments in contextual conditioning have been subject to several interpretations. The most influential has started by supposing that dissociations of freezing to a discrete conditioned stimulus (CS) for footshock versus to the context where that CS and footshock occurred are evidence for the existence of distinct representational mechanisms in the mammalian brain. Specifically, these dissociations have been interpreted to mean that activity in the hippocampal formation characterizes the operation of a configural system that records the conjunctions among the several cues (shape, appearance, smell, kinaesthetic, etc.) comprising the context or one that uses the delta rule to extract its strongest conjunctions (O'Reilly \& Rudy, 2001; Westbrook, Good, \& Kiernan, 1997). A second set of processes then forms excitatory associations between the output of this hippocampal-dependent configural system and an aversive or fear motivational system. Conditioned responding emerges from the ability of the individually sampled cues on test to retrieve or complete the configural or conjunctive representation, thereby provoking fear reactions on reexposure. Retrograde impairments in contextual conditioning and contextual discrimination emerge from a failure to rehearse, consolidate, or otherwise render permanent in memory this configural or conjunctive contextual representation.

An important implication of this view concerning the relation between contextual learning and contextual fear conditioning is that similar deficits should be apparent when amnestic manipulations are administered in advance of such conditioning. Indeed, there is evidence that manipulations able to produce retrograde 
deficits in contextual fear conditioning can also produce deficits when imposed on animals prior to conditioning. For example, lesions of the dorsal hippocampus that produce temporally bound retrograde deficits in freezing to contextual but not discrete CSs paired with footshock also produced anterograde deficits in contextual freezing (e.g., Kim, Rison, \& Fanselow, 1993; Maren \& Fanselow, 1997; Phillips \& LeDoux, 1992, 1994). Anterograde impairments in contextual fear conditioning have also been reported following adrenalectomy (Pugh, Tremblay, Fleshner, \& Rudy, 1997), administrations of glucocorticoid receptor antagonists (Pugh, Fleshner, \& Rudy, 1997), administrations of the adrenal steroid dehydroepiandrosterone (DHEA; Fleshner, Pugh, Tremblay, \& Rudy, 1997), and, indeed, conditioning at 12 noon (Rudy \& Pugh, 1998).

At least three lines of evidence have encouraged the interpretation that these anterograde deficits in contextual conditioning are caused by a failure to form a configural contextual representation. First, Fanselow and colleagues have shown that the anterograde effects of hippocampal damage on contextual conditioning can be prevented by brief exposures to the experimental context prior to hippocampal lesion (Young, Bohenek, \& Fanselow, 1994). Rudy and colleagues have reported a similar preexposure "immunization" of contextual conditioning from the deleterious effects of adrenalectomy (Pugh, Tremblay, et al., 1997), DHEA (Fleshner et al., 1997), and conditioning at midday (Rudy \& Pugh, 1998). Second, Holt and Maren (1999) as well as Westbrook et al. (1997) have shown that disruption of activity in the hippocampus or its efferents spares the extinction and latent inhibition of fear that accrue to a CS following nonreinforced exposures but abolish the context specificity of these effects. Finally, O'Reilly and Rudy (2001) arranged transport cues to signal placement in a target context. On conditioning, rats were transported in the presence of these cues and placed in a second, novel context. Rats were subject to an immediate footshock in this novel context. Consistent with previous reports, the immediate footshock failed to condition fear to the context where it was delivered (Fanselow, 1990; Kiernan \& Westbrook, 1993; but see Bevins, McPhee, Rauhut, \& Ayres, 1997). However, the immediate footshock did condition fear to the nonreinforced target context. In other words, during preexposures the rats formed a conjunctive representation of the target context and transport cues. The presence of the transport cues during conditioning enabled the animals to pattern complete from the elements (transport cues) to the conjunctive representation (the transport cues and target context). Thus, fear was conditioned to the memory of the target context that was never shocked. It is important to note that Rudy, Barrientos, and O'Reilly (2002) reported that lesions of the dorsal hippocampus impaired this conditioning to the memory representation of the preexposure context.

Nonetheless, this two-process model of contextual learning and context fear conditioning has been seriously challenged by demonstrations of intact context conditioning following hippocampal damage and by demonstrations that hippocampal damage can produce anterograde deficits in freezing to noncontextual stimuli (for review, see Gewirtz, McNish, \& Davis, 2000). One response to this challenge has been to emphasize that context fear conditioning can also proceed using a second, elemental representation system. This elemental system associates individual features comprising the contextual array with the fear motivational system
(Fanselow, 2000; Maren, 2001). Individual features of the contextual array are processed through the configural system, whereas only the elemental system is available to hippocampally damaged and adrenalectomized rats or to those injected with glucocorticoid receptor antagonists or DHEA. Through this elemental learning strategy, such rats come to fear features of the context where CS-US pairings occurred in the same way as they come to fear the CS itself. Further, the obligatory use of a common elemental system to process information about the features of the context and the CS could be expected to promote competition among these cues such that learning to fear elements of the context may overshadow learning to fear the CS and vice versa (e.g., Rescorla \& Wagner, 1972; Sutherland \& Mackintosh, 1971). Thus, treatments that produce context-specific retrograde impairments may fail to produce anterograde impairments in context fear conditioning (because the elemental system is intact) or they may produce impairments in both context and CS fear conditioning (because of reciprocal competition within the elemental system).

As noted previously, we have recently shown that that prolonged exposure to the $\mu$-opioid receptor (MOR) agonist morphine in the days to weeks following fear conditioning produces temporally bound retrograde deficits specific to contextual fear conditioning (McNally \& Westbrook, 2003). Therefore, such exposures could also be expected to produce context-specific anterograde impairments. However, as has also been noted, treatments, such as prolonged morphine exposures, that produce retrograde deficits specific to context conditioned fear may either fail to produce such deficits in the anterograde case or produce deficits in both contextand CS-conditioned fear. The experiments reported here study the effects of prolonged exposure to morphine on subsequent fear conditioning. In each experiment, rats were exposed to daily injections of morphine or saline, which terminated at varying intervals (1 to 21 days) prior to a single auditory CS (clicker)-US (footshock) pairing in a distinctive context. The conditioning that accrued to the context where CS-US pairings occurred and to the CS itself was then tested at varying intervals ( 2 min to 22 days) after conditioning. The initial experiment demonstrated that prolonged exposures to morphine produced deficits in fear conditioning to both the CS paired with footshock and the context where the pairing occurred. The remaining experiments attempted to characterize the nature of these deficits.

\section{Experiment 1}

Injection of the MOR agonist morphine for 14 days following CS-footshock pairings in a distinctive context produced retrograde deficits in contextual fear (McNally \& Westbrook, 2003). These deficits were not observed when conditioning occurred 14 or 21 days prior to the start of injections. The aims of Experiment 1 were to determine whether (a) these injections would produce anterograde amnesia for Pavlovian fear conditioning, (b) this anterograde amnesia was selective for contextual conditioning, and (c) this anterograde amnesia displayed a temporal gradient. Rats were first exposed to daily injections of saline or morphine for 14 days. They were then subjected to a single auditory CS (clicker)-footshock pairing in a distinctive context either 1,7 , or 21 days following the last injection. Finally, rats were tested for their fear reactions (freezing) to the context where conditioning occurred the day 
following conditioning and for freezing to the CS the day following context test.

\section{Method}

Subjects. The subjects were 48 experimentally naive, adult, male Wistar rats (280-350 g) obtained from a commercial supplier (Gore Hill Research Laboratories, Sydney, Australia). After arrival, the rats were housed in groups of 8 in plastic boxes $(67 \mathrm{~cm}$ length $\times 40 \mathrm{~cm}$ width $\times 22$ $\mathrm{cm}$ height) with food and water continuously available. The boxes were kept in an air-conditioned colony room maintained on a 12:12-hr light - dark cycle (lights on at 7:00 a.m.). Each rat was handled 10-30 s per day across 5 days before the start of the experiment.

Apparatus. Conditioning and CS testing were conducted in different chambers. Four identical chambers $(20 \mathrm{~cm}$ height $\times 21 \mathrm{~cm}$ length $\times 23 \mathrm{~cm}$ width) were used to footshock the rats and to test for contextually controlled freezing. The front and rear walls of these chambers as well as the hinged lid were constructed of Perspex, and the end walls were made of stainless steel. The floor in each chamber consisted of stainless steel rods, 2 $\mathrm{mm}$ in diameter, spaced $10 \mathrm{~mm}$ apart (center to center). The US was a $0.5-\mathrm{s}, 0.8-\mathrm{mA}$ unscrambled $\mathrm{AC} 50-\mathrm{Hz}$ shock from a constant-current generator that was delivered to the floor of each chamber. The current available to each floor could be adjusted using an in-line milliampere meter. Each chamber stood $5 \mathrm{~cm}$ above a tray of paper pellet bedding (Fibrecycle, Mudgeeraba, Australia) that was changed between rats. These four chambers were located within separate compartments of a wooden cabinet. The door of each compartment was kept open to permit observation of the rat. A second set of two plastic chambers $(16 \mathrm{~cm}$ height $\times 40$ $\mathrm{cm}$ length $\times 26 \mathrm{~cm}$ width) was used to test performance to the auditory CS. The front of each of these chambers was constructed of Perspex. The floor, side walls, and rear walls were made of plastic, and the roof was made of stainless steel rods. These chambers were located on the roof of the wooden cabinet that contained the footshock chambers. The discrete auditory CS consisted of a 74-dB clicker (10-Hz spike; rise time $<10 \mu \mathrm{s}$, decay time $=$ $250 \mu \mathrm{s})$ delivered from a speaker located in the ceiling of the experimental room. The background noise in the room was $69 \mathrm{~dB}$. The stimulus and background intensities were measured with a sound level meter (A scale; Type 2235, Brüel-Kjaer Instruments, Marlborough, MA) whose microphone was placed in the center of each chamber.

Procedure. The experiment involved three phases: injections of morphine or saline, fear conditioning, and testing. All rats commenced the experiment on the same day and thus differed in their ages at testing. All injections were subcutaneous in the dorsal neck region and were given in a volume of $1 \mathrm{ml} / \mathrm{kg}$, once daily. For morphine injections $(n=24)$ an ascending regime was used that results in analgesic tolerance development (Westbrook, Greeley, Nabke, \& Swinbourne, 1991). Rats were injected with $2.5 \mathrm{mg} / \mathrm{kg}$ morphine for 3 days, $5.0 \mathrm{mg} / \mathrm{kg}$ morphine for 3 days, 10.0 $\mathrm{mg} / \mathrm{kg}$ morphine for 3 days, and finally $20.0 \mathrm{mg} / \mathrm{kg}$ morphine for 5 days. For saline injections $(n=24)$, rats were injected with $0.9 \% \mathrm{w} / \mathrm{v}$ sterile saline.

Fear conditioning occurred 1,7 , or 21 days since last injection. Rats were transported in squads of 4 and placed in the conditioning chambers. One minute and $50 \mathrm{~s}$ later the 10-s CS was presented, which coterminated with delivery of footshock. Rats remained in the chambers for a further $30 \mathrm{~s}$ before being returned to their home cage.

All rats were tested for context freezing the day following conditioning and for CS freezing the day following context test. For context testing, rats were placed in the conditioning chamber, and their behavior was observed for $8 \mathrm{~min}$. For CS testing, rats were placed in the second set of chambers for $2 \mathrm{~min}$, and their behavior was observed. The CS was then presented for $3 \mathrm{~min}$ and behavior was observed. The duration of context and CS tests differed because pilot studies indicated that the levels of contextual- and CS-controlled freezing would be similar across these durations.

Data analysis. In this and subsequent experiments, performance to the context and CS on test was videotaped, and the levels of freezing were measured with a time-sampling procedure in which the rat's behavior was scored as freezing or not freezing every $2 \mathrm{~s}$. Freezing was defined as the absence of all movement except those related to breathing (Fanselow, 1980). The percentage of all samples scored as freezing was determined for each rat. Two observers, one of whom was unaware of the rat's treatment condition, scored the videotape of each rat. The unaware observer scored the rat's behavior at variable time-sampling intervals across the experiments (i.e., once every $2 \mathrm{~s}$, or $4 \mathrm{~s}$, or $5 \mathrm{~s}$ ). Thus, the scores from the aware observer were used for analysis. The agreement between these observer ratings, that is, the correlation between percentages of observations scored as freezing per rat, were high and consistently exceeded 0.9 . The data were analyzed by means of a planned orthogonal contrast testing procedure in which context and CS tests were treated as repeated measures. The Type I error rate was controlled at 0.05 for each contrast tested using the method described by Hays (1972). Four animals with a history of morphine injections died across the course of the experiment. Therefore, there were 8 animals in each of the saline groups, 7 rats in the morphine-treated groups conditioned 1 and 7 days after the last injection, and 6 rats in the morphinetreated group conditioned 21 days after last injection.

\section{Results and Discussion}

The left panel of Figure 1 shows the mean and the standard error of the mean (SEM) levels of freezing during the context test conducted the day after conditioning. Inspection of the panel indicates a linear increase in freezing across the interval since last injection such that rats conditioned 1 day after last injection of morphine displayed less freezing to the context as compared with saline controls, whereas rats conditioned 21 days after the last injection of morphine did not differ from saline controls.

The right panel of Figure 1 shows the mean and SEM levels of freezing during the CS test conducted the day after context test. There were minimal levels of pre-CS freezing (morphine group: 1 -day mean $=1 \%, S E M=1$; saline group: 1 -day mean $=3 \%$, $S E M=2$; morphine group: 7-day mean $=4 \%, S E M=3$; saline group: 7-day mean $=17 \%, S E M=10$; morphine group: 21 -day mean $=3 \%, S E M=2$; saline group: 21 -day mean $=9 \%, S E M=$ $5)$. Inspection of the panel indicates that rats conditioned after a

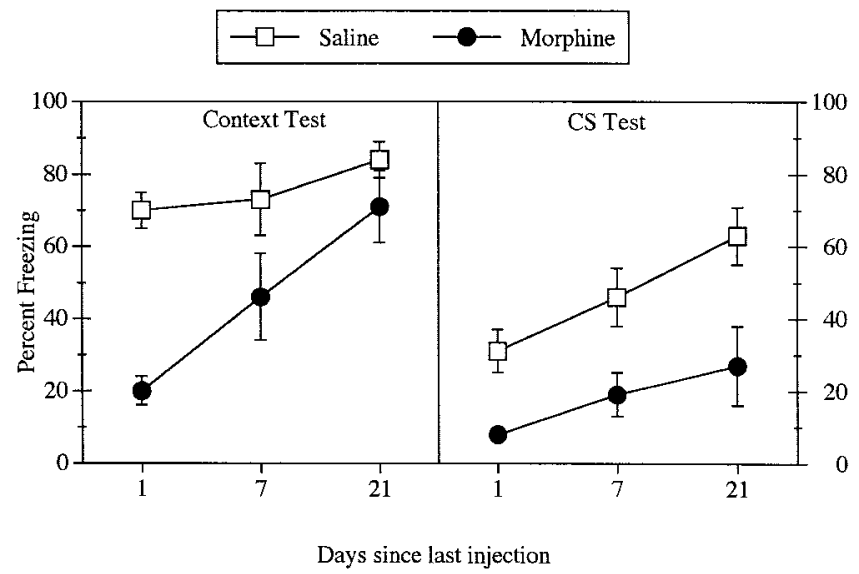

Figure 1. Mean and SEM levels of freezing on context test (left panel) and CS test (right panel) in Experiment 1. One day, 7 days, or 21 days following 14 days of injection of morphine or saline, rats were subject to a single CS-footshock pairing in a distinctive context. Rats were tested for freezing to the context the day after conditioning and for freezing to the CS the day after context test. $\mathrm{CS}=$ conditioned stimulus 
history of morphine injections displayed less freezing to the CS than rats conditioned after an equivalent history of saline injections. The panel also indicates a linear increase in freezing across the interval since last injection such that rats conditioned 1 day after last injection of morphine or saline displayed less freezing to the CS as compared with rats conditioned 21 days after the last injection of morphine or saline. However, there appeared to be no interaction between these two results.

The statistical analysis confirmed these observations. There was an overall main effect for morphine versus saline, $F(1,38)=36.2$, $p<.01$, such that there was significantly less freezing among rats with a history of morphine injections as compared with the saline controls. There was also an overall main effect for the interval between last injection and test, $F(1,38)=17.6, p<.01$, such that there was linear increase in freezing across this interval between last injection and test. There was, however, no overall interaction, $F(1,38)<1, p>.05$.

There was a significant main effect for test type, $F(1$, $38)=21.2, p<.01$, such that there were significantly greater levels of freezing on context test than CS test. It is important to note that the deficit in conditioning produced by the history of morphine injections was nonselective because this deficit failed to interact with test type, $F(1,38)=3.2, p>.05$. The linear increase in freezing as a function of the interval between last injection and test also did not interact with test type, $F(1,38)<1, p>.05$. However, the three-way interaction was significant, $F(1$, $38)=14.7, p<.01$. This interaction indicates that, although freezing did increase as a linear function of time since last injection, the difference in this increase between rats with a history of morphine and saline was greater for the context test than the CS test. In other words, the effects of time were more pronounced in the morphine than saline rats especially in the context test.

This experiment has shown that a history of morphine injections produces anterograde impairments in Pavlovian fear conditioning. Specifically, conditioning to the context where CS-US pairings occurred was impaired 1 day but not 21 days after last exposure to morphine, whereas conditioning to the CS was equally impaired 1 day and 21 days after last exposure to morphine. The overall linear increase in freezing levels on the CS and context test is worthy of comment. Rats in this experiment were handled daily up to the conditioning episode but were not handled across the retention interval. Thus, it is possible that the increase in freezing across the interval between injection and conditioning emerged because of a loss of the latent inhibition that may have accrued to the handling cues (e.g., Killcross, Kiernan, Dwyer, \& Westbrook, 1998). Irrespective of why freezing increased across this interval, the recovery from morphine-induced deficits in contextual conditioning was observed over and above this increase.

\section{Experiment 2}

The results of Experiment 1 showed that repeated injections of morphine produced anterograde deficits in auditory CS fear conditioning when the interval between injections and conditioning was short (1 day) or long (21 days). Such exposures also produced deficits in context fear conditioning when this interval was short but not when it was long. These results suggest that a history of morphine provokes a transient impairment in context fear conditioning and a more permanent impairment in CS conditioning.
According to this suggestion, the critical interval for these effects was that between the termination of morphine exposures and fear conditioning, which was either 1 or 21 days. However, the design used in that experiment confounded this interval with that between termination of morphine exposures and test. That is, rats were not just subjected to a short interval (1 day) between morphine exposures and conditioning; they were also subjected to a short interval (2-3 days) between those exposures and test. Likewise, rats were not just subjected to a long interval (21 days) between morphine exposures and conditioning; they were also subjected to a long interval (22-23 days) between those exposures and test.

This concern can be made explicit. One interpretation of the results obtained in Experiment 1 begins by supposing that the levels of context conditioning are greater than those of CS conditioning. It then supposes that the termination of morphine exposures provokes withdrawal whose severity decreases across time. Further, a critical component of withdrawal is hyperactivity. According to this interpretation, therefore, the deficits present when rats were conditioned and tested shortly after termination of morphine is a consequence of interference between hyperactivity and the target response (freezing). The differential recovery of contextand CS-controlled freezing when rats were tested some time after termination of morphine is due to alleviation of withdrawal and its associated hyperactivity. However, although alleviated, the remaining withdrawal is able to interfere with freezing to the less strongly conditioned CS.

The aim of Experiment 2 was to study this interpretation. The design was a $2 \times 2$ factorial where the first factor was type of drug history (morphine vs. saline) and the second factor was the length of the retention interval interpolated between conditioning and test (1 day vs. 21 days). If the deficits observed in the previous experiment were due to variations across time in the levels of withdrawal and its associated hyperactivity, a similar pattern of results will be observed here. That is, rats tested shortly after termination of morphine exposures will exhibit less freezing (they are more hyperactive) than those tested some time after termination of those exposures. Further, these differences between rats tested shortly or some time after termination of morphine should be greater in the case of context- than CS-controlled freezing.

\section{Method}

Subjects and apparatus. The subjects were 32 experimentally naive rats $(280-350 \mathrm{~g})$ of the same sex and strain and obtained from the same source as in Experiment 1. They were kept under the conditions reported previously. Each rat was handled $10-30$ s per day across 5 days before the start of the experiment and on each day of the experiment. The apparatus was that used in Experiment 1.

Procedure. The experiment involved three phases: morphine or saline injections, fear conditioning, and testing. Rats received daily injections of morphine $(n=16)$ or saline $(n=16)$ as described for Experiment 1 . Fear conditioning occurred the day after the last injection and was identical to that used in Experiment 1. Rats were tested for context freezing either 1 day or 21 days following conditioning. The test for CS-elicited freezing took place the day after context test (i.e., 2 days or 22 days following conditioning).

\section{Results and Discussion}

The left panel of Figure 2 shows the mean and the SEM levels of freezing during the context test conducted either 1 day or 21 


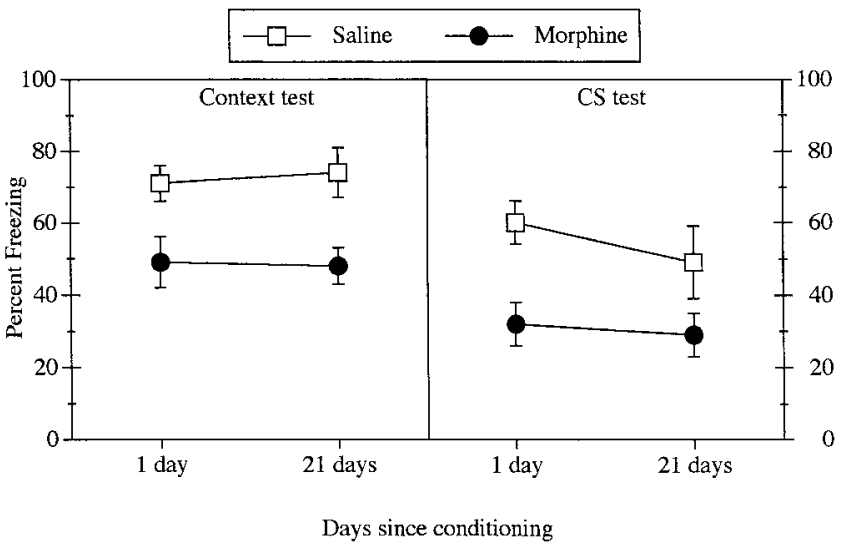

Figure 2. Mean and SEM levels of freezing on context test (left panel) and CS test (right panel) in Experiment 2. One day following 14 days of injection of morphine or saline, rats were subject to a single CS-footshock pairing in a distinctive context. They were tested for freezing to the context either 1 or 21 days after conditioning and for freezing to the CS the day after context test. $\mathrm{CS}=$ conditioned stimulus.

days after conditioning. Inspection of the panel indicates that rats with a history of morphine injections displayed less freezing to the context where CS-US pairings occurred than rats with a history of saline injections. Critically, this deficit did not recover across the retention interval.

The right panel of Figure 2 shows the mean and SEM levels of freezing during the CS test conducted the day after context test. The levels of pre-CS freezing were higher in this than the previous experiment (morphine group: 1-day mean $=20 \%, S E M=5$; saline group: 1 -day mean $=42 \%, S E M=7$; morphine group: 21-day mean $=13 \%, S E M=5$; saline group: 21 -day mean $=$ $34 \%, S E M=10)$. Inspection of the panel indicates that rats with a history of morphine injections displayed less freezing to the CS than rats with a history of saline injections. Again, this deficit did not recover across the retention interval.

These observations were confirmed by the statistical analysis. There was an overall main effect for drug history, $F(1,28)=25.8$, $p<.01$, such that rats with a history of morphine injections displayed less freezing than rats with a history of saline injection. There was no overall main effect for retention interval, $F(1$, $28)<1, p>.05$. There was also no overall interaction between drug history and retention interval, $F(1,28)<1, p>.05$. There was no overall main effect for text type, $F(1,28)<1, p>.05$, such that levels of freezing observed on context and CS test did not differ. There were no interactions between drug history, $F(1$, $28)<1, p>.05$, or retention interval, $F(1,28)=1.2, p>.05$, and test type. Finally, there was no three-way interaction, $F(1,28)<1$, $p>.05$.

This experiment has confirmed that a history of morphine injections produces an anterograde deficit in Pavlovian fear conditioning when rats are conditioned 1 day after last exposure to morphine. This deficit was nonselective for the contents of the learning established during conditioning: Rats with a history of morphine injections were impaired in both contextual and CS conditioning. It is important to note that the experiment has shown that this deficit is not alleviated by interpolation of a retention interval between conditioning and test. This failure to observe recovery of context or CS-controlled freezing across the retention interval suggests that the critical variable for the deficits in fear conditioning is the interval between the termination of morphine exposures and conditioning rather than the interval between termination of those exposures and test. In Experiment 1, morphinetreated rats displayed similar and high levels of context freezing to saline controls when conditioned 21 days after last injection and tested the following days, whereas the rats in the present experiment, tested at the same interval since last injection, displayed significantly less freezing than saline controls.

\section{Experiment 3}

Two lines of evidence indicate that performance on tests for conditioned fear in the seconds to minutes following conditioning can be dissociated from the performance observed in the hours to days following conditioning. The first is that the fear observed 1 day after conditioning can be impaired, whereas the fear observed immediately after conditioning remains intact. Manipulations that produce these impairments in long-term but not short-term fear conditioning include dorsal hippocampal stimulation (e.g., Kesner \& Connor, 1972), hippocampal lesion (e.g., Kim et al., 1993), antagonism of $N$-methyl-D-aspartate receptors (e.g., Kim, Fanselow, DeCola, \& Landeira-Fernandez, 1992), time of day that conditioning occurs (Rudy \& Pugh, 1998), adrenalectomy (Pugh Tremblay, et al., 1997), and targeted deletion of the gene encoding the cyclic-AMP response element binding protein (CREB; Bourtchuladze et al., 1994). The second line of evidence is that fear varies as a nonmonotonic function of the retention interval between conditioning and test. This function is characterized by high levels of performance immediately after conditioning, which decreases in the hours following conditioning only to recover $24 \mathrm{hr}$ after conditioning (Kamin, 1957). The nadir of this function is variable such that increases in conditioning shift the function to the left (Brush, 1971).

It follows from these dissociations of short-term from long-term fear that the anterograde deficits observed in Experiments 1 and 2 could have emerged from a failure to encode the relevant associations in a short-term store or from a failure to consolidate these associations in a long-term store. The aim of Experiment 3 was to distinguish between these possibilities. The design was a $2 \times$ $3 \times 2$ factorial. The first factor was drug history (morphine vs. saline). The second factor was when rats were tested ( 2 min vs. 6 hr vs. $24 \mathrm{hr}$ ). The third factor was the type of test (context vs. CS). In contrast to the previous experiments reported here, test type was a between-subjects factor in this experiment. This was necessary because of the need to test animals 2 min after conditioning for performance to the CS and context. If the history of morphine injections acted simply to impair encoding of the relevant associations in some short-term store, morphine-treated rats should display less fear than saline-treated rats at all time points. However, if the history of morphine injections acted selectively to impair encoding of the relevant associations in a long-term store, morphine-treated rats should display equivalent levels of freezing to saline-treated rats when tested $2 \mathrm{~min}$ and $6 \mathrm{hr}$ after conditioning but should display less fear when tested $24 \mathrm{hr}$ after conditioning. 


\section{Method}

Subjects and apparatus. The subjects were 96 experimentally naive rats $(280-350 \mathrm{~g})$ of the same sex and strain and obtained from the same source as in Experiment 2. They were kept under the conditions reported previously. Each rat was handled 10-30 s per day across 5 days before the start of the experiment and on each day of the experiment. The apparatus was that used in Experiment 1.

Procedure. The experiment involved three phases: morphine or saline injections, fear conditioning, and testing. Rats received daily injections of morphine $(n=48)$ or saline $(n=48)$ as described for Experiment 1 . Fear conditioning occurred the day after last injection and was identical to that used in Experiment 1. Half of the rats in each drug condition were tested for context freezing. This test took place either $2 \min (n=8$ per drug condition), $6 \mathrm{hr}$ ( $n=8$ per drug condition), or $24 \mathrm{hr}$ ( $n=8$ per drug condition) following conditioning. The remaining rats in each drug condition were tested for CS freezing. This test took place $2 \min (n=8$ per drug condition), $6 \mathrm{hr}$ ( $n=8$ per drug condition), or $24 \mathrm{hr}(n=8$ per drug condition) following conditioning. It is worth noting that conditioning occurred at 8 a.m. Thus, in addition to differing in terms of retention interval, the rats in this experiment also differed in terms of the time of day that they were tested ( 8 a.m. vs. 2 p.m.). The available data indicate that conditioning rats in the early afternoon (12 p.m.) can have an influence on the acquisition of contextual fear conditioning but does not affect the expression of that fear conditioning (Rudy \& Pugh, 1998). It is unlikely that any freezing among rats tested 2 min after conditioning represents an unconditioned response to footshock. Fanselow has provided two lines of evidence that the freezing observed shortly (minutes) after footshock is a conditioned response. First, when rats are tested shortly after footshock, levels of freezing are significantly higher among rats tested in the same context where the footshock was delivered as opposed to a second, novel context (Fanselow, 1980). Second, freezing is not observed when footshock is delivered shortly after placement in the conditioning chamber (Fanselow, 1986).

Data analysis. The data were analyzed by means of a planned orthogonal contrast testing procedure in which the influence of drug history (morphine vs. saline) and test type (context vs. CS) were analyzed at each conditioning to test interval $(2 \mathrm{~min}, 6 \mathrm{hr}$, and $24 \mathrm{hr})$.

\section{Results and Discussion}

The mean and the SEM levels of freezing on context test are shown in the left panel of Figure 3. Inspection of the panel indicates that freezing to the context was a nonmonotonic function of the retention interval: Testing rats either 2 min or $24 \mathrm{hr}$ after conditioning resulted in high levels of freezing, whereas testing rats $6 \mathrm{hr}$ after conditioning resulted in lower levels of freezing. The largest difference in levels of freezing between rats with a history of saline and morphine injections appeared to be at $24 \mathrm{hr}$.

The mean and SEM levels of freezing on CS test are shown in the right panel of Figure 3. The levels of pre-CS freezing were variable and similar to those observed in Experiments 1 and 2 (morphine group: 2 -min mean $=22 \%, S E M=7$; morphine group: $6-\mathrm{hr}$ mean $=6 \%, S E M=2$; morphine group: $24-\mathrm{hr}$ mean $=22 \%$, $S E M=8$; saline group: 2 -min mean $=30 \%, S E M=6$; saline group: 6 -hr mean $=8 \%, S E M=4$; saline group: 24 -hr mean $=$ $24 \%, S E M=11)$. Inspection of the panel indicates that the function relating freezing to the retention interval differed from the nonmonotonic function observed on context test and that the influence of the history of morphine injections was reduced.

These observations were confirmed, at least in part, by the statistical analysis. There was no overall main effect for test type, $F(1,84)=1.0, p>.05$, such that levels of freezing to the context

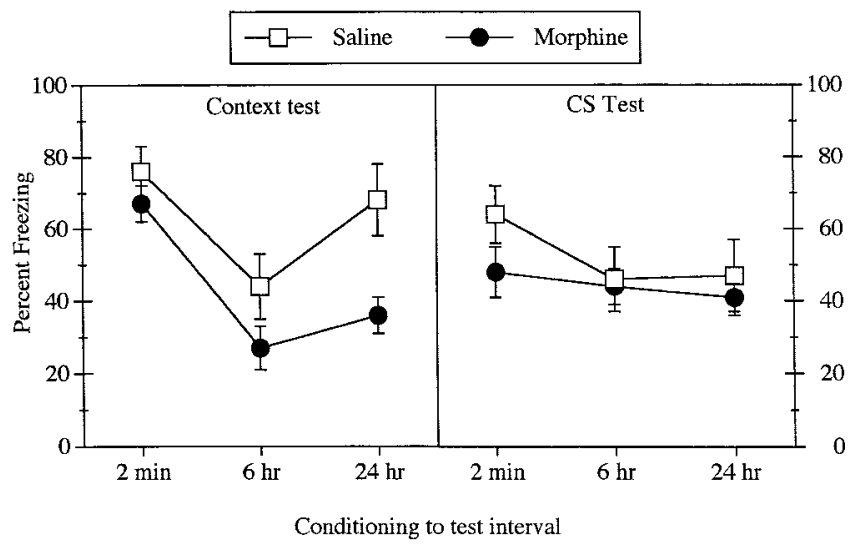

Figure 3. Mean and SEM levels of freezing on context test (left panel) and CS test (right panel) in Experiment 3. One day following 14 days of injection of morphine or saline, rats were subject to a single CS-footshock pairing in a distinctive context. They were tested for freezing to the context or to the CS either $2 \mathrm{~min}, 6 \mathrm{hr}$, or $24 \mathrm{hr}$ after conditioning. CS = conditioned stimulus.

did not differ significantly from freezing to the CS. There was no effect for drug history on the tests conducted $2 \mathrm{~min}, F(1$, $84)=2.5, p>.05$, or $6 \mathrm{hr}, F(1,84)=1.5, p>.05$, after fear conditioning. However, there was a significant effect of drug history $24 \mathrm{hr}$ after conditioning, $F(1,84)=6.7, p=.01$. This indicates that, averaged across test type, rats with a history of morphine injections displayed less freezing than rats with a history of saline injections. However, there were no two-way interactions between the influence of drug history (morphine vs. saline) and test type (context vs. CS) at any time interval, all $F s(1,84)<2.8$, $p>.05$. These results indicate that the anterograde deficits in conditioning produced by the history of morphine injections were only apparent when rats were tested $24 \mathrm{hr}$ after conditioning but were not significantly different between context and CS tests.

The absence of a significant two-way interaction between morphine versus saline history and context versus CS test at the 24-hr test is surprising. Inspection of Figure 3 suggests little difference between morphine-treated and saline-treated rats on CS test. That is, inspection of Figure 3 suggests that the anterograde deficit produced by morphine was restricted to rats tested for contextual fear $24 \mathrm{hr}$ after conditioning and was not apparent among rats tested for CS-elicited fear $24 \mathrm{hr}$ after conditioning. The statistical analysis failed to confirm this observation. However $t$ tests, based on simple comparison between morphine- and saline-treated rats tested $24 \mathrm{hr}$ after conditioning, revealed a significant difference (i.e., anterograde deficits) for context test ( $p=.01)$ but not CS test $(p>.05)$. The failure of the $2 \times 2$ interaction term to approach statistical significance at the $24-\mathrm{hr}$ test tempers speculation regarding potential differences between the two tests. Nonetheless it is worth noting that one important difference between this experiment and the previous experiments reported here was that rats were tested for fear reactions to the CS in the absence of any prior extinction of the fear that had accrued to the conditioning context (see General Discussion).

This experiment has confirmed that a history of morphine injections produces an anterograde impairment in Pavlovian fear 
conditioning when rats are conditioned 1 day after last injection and tested 1 day after conditioning. However, this anterograde impairment was absent when rats were tested either 2 min or $6 \mathrm{hr}$ after conditioning. This absence could not simply be due to ceiling or floor effects obscuring differences because freezing varied as a nonmonotonic function of retention interval, that is, freezing displayed the Kamin (1957) effect. In sum, the results from this experiment are consistent with effects of hippocampal lesions, administrations of $\mathrm{N}$-methyl-D-aspartate receptor antagonists, and targeted disruption of the CREB gene among other manipulations and suggest that the history of morphine injections preserved short-term but impaired long-term fear conditioning.

\section{Experiment 4}

Experiments 1-3 show that a history of morphine injections produces deficits in Pavlovian fear conditioning. These deficits were not reliably selective with respect to the modality of the conditioned stimulus such that impairments in conditioning to both the contextual cues and the auditory CS were observed. These deficits required more than a 6-hr interval between conditioning and test for their appearance. These deficits also do not appear because of an inability of the morphine-treated rats to display the target response (freezing). One interpretation of these results could emphasize that the exposures to morphine interfered with the consolidation of fear memories in some long-term memory store. There is, however, another plausible interpretation of these results. The designs used in Experiments 1-3 necessarily entailed the compound conditioning of contextual and auditory cues. Under such circumstances, the amount of conditioning that accrues to each element of the stimulus compound can be less than would have accrued to each element conditioned in isolation. For example, James and Wagner (1980), using a conditioned suppression preparation, reported that rats displayed less conditioning to a light or tone CS when given a single compound conditioning trial as compared with when either element was conditioned in isolation. Mackintosh and colleagues (e.g., Kaye, Gambini, \& Mackintosh, 1988; Mackintosh \& Reese, 1979) have reported similar evidence for one-trial overshadowing. The interpretation favored by these authors emphasized that one-trial overshadowing, when detected, emerges from competition for a finite amount of attentional or working-memory resources. According to this line of reasoning, the pattern of responding observed in the present experiments emerged not because the history of morphine injections impaired the consolidation of fear memories but instead because these injections increased susceptibility to one-trial overshadowing.

These two interpretations offer different predictions regarding the influence of omitting the auditory CS and/or varying CS intensity. Interpretations that emphasize a role for impairments in memory consolidation appear to predict that deficits in freezing should be observed regardless of the presence versus absence of the CS as well as variations in CS intensity. By contrast, an interpretation that emphasizes a role for one-trial overshadowing in producing the deficits observed in Experiments 1-3 predicts that the anterograde deficits produced by morphine should be alleviated by omitting the CS and could also be influenced by variations in CS intensity. Experiment 4 used a $2 \times 3$ factorial design to test these possibilities. The first factor was drug history (saline vs. morphine). The second factor was intensity of the auditory CS (0
dB vs. $70 \mathrm{~dB}$ vs. $82 \mathrm{~dB}$ ). In this experiment, rats were pretreated with morphine or saline. Conditioning occurred the day following last injection. All rats were tested for fear reactions to the conditioning context the day after conditioning and for fear reactions to the auditory CS the day after context test.

\section{Method}

Subjects and apparatus. The subjects were 48 experimentally naive rats $(280-350 \mathrm{~g})$ of the same sex and strain and obtained from the same source as in Experiment 1. Each rat was handled 10-30 s per day across 5 days before the start of the experiment and on each day of the experiment. They were kept under the conditions reported previously. The apparatus was that used in Experiment 1.

Procedure. The experiment involved three phases: morphine or saline injections, fear conditioning, and testing. Rats received daily injections of morphine $(n=24)$ or saline $(n=24)$ as described for Experiment 1 . Fear conditioning was similar to that used in Experiment 1 with the following variations. Conditioning took place 1 day after last injection. Rats in the 0 - $\mathrm{dB}$ groups were conditioned in the absence of the auditory CS. Rats in the $70-\mathrm{dB}$ group were conditioned with a 70-dB auditory CS. Rats in the $82-\mathrm{dB}$ group were conditioned with an $82-\mathrm{dB}$ auditory $\mathrm{CS}$. Rats were tested for context freezing 1 day following conditioning. The test for CS-elicited freezing took place the day after context test. Rats in the 70-dB and 82-dB groups were tested with the same intensity clicker as was used during conditioning. Half of the rats in the $0-\mathrm{dB}$ group were tested with the $70-\mathrm{dB}$ clicker, whereas the remainder were tested with the $82-\mathrm{dB}$ clicker. There were no differences in freezing between rats in the 0 - $\mathrm{dB}$ group tested with the $70-\mathrm{dB}$ clicker versus the $82-\mathrm{dB}$ clicker, thus the data for this group was collapsed across this manipulation. Two rats with a history of morphine injections died during dependence induction. One rat with a history of morphine injections developed an infection and was not included in the experiment, and 1 rat in the $82-\mathrm{dB}$ saline group was excluded because of a failure of the footshock generator. Therefore, there were 8 rats in the $0-\mathrm{dB}$ saline group and the $70-\mathrm{dB}$ saline group, and there were 7 rats in each of the remaining groups.

\section{Results and Discussion}

The mean and SEM levels of freezing on context test are shown in the left panel of Figure 4. The mean and SEM levels of freezing

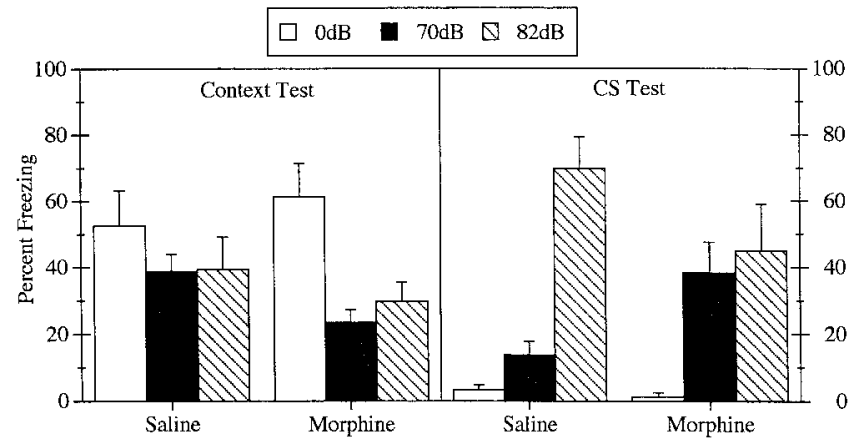

Figure 4. Mean and SEM levels of freezing on context test (left panel) and CS test (right panel) in Experiment 4. One day following 14 days of injection of morphine or saline, rats were subject to a single CS-footshock pairing in a distinctive context. The CS intensity was $0 \mathrm{~dB}, 70 \mathrm{db}$, or $82 \mathrm{db}$ Rats were tested for freezing to the context the day after conditioning and for freezing to the CS the day after context test. CS = conditioned stimulus. 
on CS test are shown in the right panel of Figure 4. The levels of pre-CS freezing were variable and similar to those observed in Experiments 1 and $2(0-\mathrm{dB}$ morphine group: mean $=11 \%, S E M=$ 5; 70-dB morphine group: mean $=7 \%, S E M=2 ; 82-\mathrm{dB}$ morphine group: mean $=6 \%, S E M=2 ; 0-\mathrm{dB}$ saline group: mean $=8 \%$, $S E M=4 ; 70-\mathrm{dB}$ saline group: mean $=3 \%, S E M=1 ; 82-\mathrm{dB}$ saline group: mean $=8 \%, S E M=4)$.

Inspection of the panels suggests two patterns of responding. Among saline-treated rats, there was little evidence for one-trial overshadowing: Freezing to the context was relatively stable across variations in CS intensity, whereas freezing to the CS increased as a function of its intensity. Among morphine-treated rats, there was no evidence for deficits in contextual freezing among rats conditioned in the absence of the CS, but there were such deficits in contextual freezing among rats conditioned with either the 70 - or $82-\mathrm{dB}$ CS. Moreover, there appeared to be enhanced freezing to the 70-dB CS among morphine-treated rats but reduced conditioning to the $82-\mathrm{dB}$ CS

The analysis confirmed these observations. There was no overall main effect for injection type (morphine vs. saline), $F(1,38)<1$, $p>.05$. There was no overall difference in freezing between rats conditioned with the $0-\mathrm{dB}$ clicker and rats conditioned with the 70 -db clicker, $F(1,38)<1, p>.05$. There was an overall difference in freezing among rats conditioned with the $70-\mathrm{dB}$ clicker and rats conditioned with the 82 -dB clicker, $F(1,38)=7.7$, $p<.03$. There were no overall interactions between injection type and CS intensity, $F(1,38)=3.1$ and $\langle 1, p\rangle .05$. There was an overall difference in levels of freezing between the context and CS tests, $F(1,38)=13.3, p<.01$.

The difference in freezing between context and CS tests did not interact with type of injection, $F(1,38)<1, p>.05$. However, this difference did interact with the increase in CS intensity from 0 $\mathrm{dB}$ to $70 \mathrm{~dB}$, indicating the presence of overshadowing such that the increase in freezing observed between the $0-\mathrm{dB}$ and $70-\mathrm{dB}$ clicker was associated with a reduction in freezing to the context, $F(1,38)=36.2, p<.01$. A similar interaction was observed between the increase in CS intensity from $70 \mathrm{~dB}$ to $82 \mathrm{~dB}$ and context versus CS test, again indicating that the increase in freezing observed between the 70- $\mathrm{dB}$ and $82-\mathrm{dB}$ clicker was associated with a reduction in freezing to the context, $F(1,38)=11.2, p<$ .01. Critically, each of these interactions of CS intensity and context versus CS test interacted with drug history. Thus, there was a three-way interaction between injection type, the 0-dB versus 70-dB CS, and context versus CS test, $F(1,38)=9.5, p<$ .01. From inspection, morphine-treated rats did not differ from saline-treated rats in contextual freezing at the 0 - $\mathrm{dB}$ clicker, and the presence of the 70-dB clicker was associated with less contextual freezing but more CS freezing among morphine-treated rats. There was also a three-way interaction between injection type, the increase in CS intensity from $70 \mathrm{~dB}$ to $82 \mathrm{~dB}$, and context versus $\mathrm{CS}$ test, $F(1,38)=10.7, p<.01$. From inspection, the increase in CS intensity from $70 \mathrm{db}$ to $82 \mathrm{~dB}$ was associated with a greater increase in CS freezing among saline-treated rats than morphinetreated rats.

Post hoc contrasts were tested, using the method described by Rodger (1967), to study these three-way interactions and confirm these conclusions. Regarding performance on context test, salinetreated rats conditioned with the $0-\mathrm{dB} C S$ did not differ from saline-treated rats conditioned with the 70-dB and 82-dB CS, F(1,
$38)=2.2, p>.05$, whereas morphine-treated rats conditioned with the $0-\mathrm{dB}$ CS did differ significantly from morphine-treated rats conditioned with the $70-\mathrm{dB}$ and $82-\mathrm{dB} \mathrm{CS}, F(1,38)=13.3$, $p<.01$. In other words, the auditory CS failed to overshadow contextual conditioning among saline-treated rats but did overshadow contextual conditioning among morphine-treated rats. Regarding performance on CS test: Morphine-treated rats showed more freezing to the $70-\mathrm{dB} \mathrm{CS}$ than saline-treated rats, $F(1$, $38)=5.9, p<.05$, but less freezing to the 82-dB CS than saline-treated rats, $F(1,38)=5.6, p<.05$.

The first important result from this experiment is that the anterograde amnestic effects of a history of morphine injections depend on the presence of an auditory CS. Rats with a history of morphine injections conditioned in the absence of the auditory CS did not differ in levels of context freezing from rats with a history of saline injections. The consequence of introducing the auditory CS was to selectively impair contextual conditioning among morphine-treated rats. In other words, there was no evidence for one-trial overshadowing of context by CS among saline-treated rats (Rescorla \& Wagner, 1972), but there was evidence for such one-trial overshadowing among morphine-treated rats such that conditioning to the context suffered as a consequence of the presence of the CS (James \& Wagner, 1980; Pearce, 1994; Sutherland \& Mackintosh, 1971). This interaction of deficits in context conditioning with the presence versus absence of the CS is inconsistent with the interpretation that was encouraged by the results of Experiments 1-3. Namely, this interaction is inconsistent with an interpretation that asserts that a history of morphine injections produced anterograde impairments in fear conditioning by disrupting the consolidation of fear memories.

The second important result from this experiment is that the previously identified deficit in conditioning to the CS produced by a history of morphine injections depended on CS intensity: Rats with a history of morphine injections and conditioned with a low-intensity CS showed enhanced CS-elicited freezing relative to saline controls; in contrast, when these rats were conditioned with a high-intensity CS they showed less freezing than saline controls. In other words, the morphine-treated rats conditioned with the 82-dB CS were similar to their counterparts in the previous experiments conditioned with the 74-dB CS. However, the morphine-treated rats conditioned with the low-intensity, 70-dB CS were different from their counterparts in the previous experiments conditioned with a 74-dB CS, in that rats conditioned with the lower intensity CS showed reduced conditioning to the context but enhanced conditioning to the CS.

\section{General Discussion}

Damage to the dorsal hippocampus (Maren et al., 1997), adrenalectomy (Pugh, Tremblay, et al., 1997), administration of glucocorticoid receptor antagonists or DHEA (Fleshner et al., 1997; Pugh, Fleshner, et al., 1997), and conditioning at 12 noon (Rudy \& Pugh, 1998), among other manipulations, produce anterograde deficits in contextual fear conditioning. The explanation typically offered for these deficits is that they reflect failures in consolidating contextual fear representations in memory. These representations are derived from detecting the conjunctions among the several cues (shape, appearance, smell, kinaesthetic, etc.) comprising the context (O'Reilly \& Rudy, 2001) or from exploiting the delta 
rule to extract the strongest of these conjunctions (McLaren, Kaye, \& Mackintosh, 1989; Westbrook et al., 1997). The experiments reported here studied whether a regime of morphine injections known to produce a temporally bound and modality-specific retrograde amnesia for Pavlovian contextual fear conditioning (McNally \& Westbrook, 2003) would also produce anterograde amnesia for that conditioning. The results of Experiment 1 showed that the history of morphine injections produced anterograde amnesia for fear conditioning, which was initially nonselective for the context versus CS but was eventually selective in disrupting conditioning to the CS when conditioning took place 21 days after last injection. The results of Experiment 2 showed that these deficits could not be attributed simply to performance deficits because freezing did not recover across a retention interval interpolated between conditioning and test. The results of Experiment 3 showed that these deficits required a greater than 6-hr interval between conditioning and test for their manifestation.

Taken together the results of Experiments 1-3 are consistent with the notion that anterograde amnesia for fear conditioning emerged because of deficits in consolidation of fear memories. However, the results of Experiment 4 appear inconsistent with this interpretation. Experiment 4 showed that the deficit in fear conditioning depended on the presence of an auditory CS. Rats with a history of morphine injections conditioned in the absence of an auditory CS did not differ in levels of contextual freezing from control rats with a history of saline injections. Deficits in contextual conditioning were only observed following the introduction of the CS.

One interpretation of this interaction between the presence of the auditory CS and deficits in contextual conditioning invokes the existence of discrete representational mechanisms. The models reviewed in the introduction emphasize that in otherwise intact rats, individual contextual elements are processed in a conjunctive or configural representational system that gives rise to an integrated contextual representation that is then associated with footshock. The auditory CS, on the other hand, is processed within an elemental representational system and then associated with footshock. Interference with the configural representational system results in processing of the individual features of the contextual array within the elemental representational system. These models suggest that saline-treated animals represented the contextual cues using the configural system operating independently of the elemental system deployed to represent the CS. The chronic exposures to morphine impaired this configural system. Morphinetreated rats conditioned with the unsignaled US still developed substantial levels of context fear because they used the elemental system to associate discrete contextual features with the US. Morphine-treated rats conditioned with the signaled footshock acquired less fear because the conjoint processing of contextual features and the discrete CS in the elemental system may have increased the interference between them.

A second interpretation rejects the existence of such distinct representational systems. According to the Rescorla-Wagner (1972) model of Pavlovian conditioning, overshadowing is a multitrial phenomenon, emerging across trials from competition between CSs for a finite amount of associative strength. This competition does not occur on the first conditioning trial and so associative strength is distributed to each element of the compound CS (e.g., context and auditory CS) in direct proportion to the salience of each and independently of that distributed to the other. The performance of saline-treated rats in the experiments reported here falls neatly from this model. Although the Rescorla-Wagner model fails to explain the performance of the morphine-treated rats in Experiment 4, any model that accounts for one-trial overshadowing of the context by the CS can explain this performance. For example, one view emphasizes that one-trial overshadowing is a consequence of competition for limited attentional (e.g., Sutherland \& Mackintosh, 1971) or working-memory (Wagner, 1981) resources. Moreover, the Rescorla-Wagner model can be modified to account for such one-trial effects by supposing that the alpha value of the context is regulated by the alpha value of the CS. That is, $\Delta \mathrm{V}$ context $=\left(\alpha_{\text {context }} / \alpha_{\text {context }}+\alpha_{\mathrm{CS}}\right) \times(\lambda-\mathrm{VX})$ (see Pearce, 1994). According to this line of reasoning, anterograde deficits in contextual fear conditioning are to be understood by specifying why saline-treated rats should behave in a manner consistent with the Rescorla-Wagner learning rule, whereas morphine-treated animals behave in a manner consistent with the modifications to this rule offered by Pearce (1994). Although explaining the deficits in context learning, these accounts might not offer adequate explanations of the deficits in CS learning observed here. It could be suggested that the deficits in CS and context conditioning reported here simply represented reciprocal one-trial overshadowing of each by the other. However, two findings suggest that the deficits in performance to the CS may have emerged for reasons different to the deficits in contextual freezing. First, Experiment 1 showed that deficits in context conditioning displayed a temporal gradient, whereas the deficit in CS conditioning did not. Second, Experiment 4 revealed that the amount of overshadowing of context by CS among morphine-treated rats was similar for the $70-\mathrm{dB}$ and $82-\mathrm{dB}$ CS. The amount of freezing elicited by the 70-dB and $82-\mathrm{dB}$ CS was also very similar among morphine- but not saline-treated rats. In other words, although clearly sensitive to the presence versus absence of the CS, there was no evidence here that morphine-treated rats were sensitive to variations in CS intensity. These results could be interpreted to mean that the history of morphine injections produced a longlasting impairment in sensory processing of the CS.

Alternatively, it might be suggested that the deficits in freezing to the CS emerged from the practice of testing rats for fear reactions to the context and then to the $\mathrm{CS}$. We used this serial test procedure in order to aid comparison with the existing literature on anterograde amnesia for Pavlovian fear conditioning and with the results obtained when prolonged exposures to morphine followed fear conditioning (McNally \& Westbrook, 2003). In this latter case, prior context testing among rats subjected to postconditioning exposures to morphine did not alter freezing to the CS compared with rats subjected to postconditioning exposures to saline. The results of Experiment 4 reported here also demonstrate that deficits in CS freezing are not inevitable consequences of the practice of testing rats first for context freezing. In that experiment, rats with a history of morphine injections tested for fear to the low intensity CS showed significantly enhanced CS-elicited freezing as compared with saline controls. Nevertheless, it remains possible that the initial context test made some contribution to the reduced CS freezing observed among morphine-treated rats. The results of Experiment 3 are especially interesting in this regard because rats in that experiment were tested for fear reactions to only the context 
or the CS, and there was little impairment in CS freezing. Further examination of this issue is worthwhile.

Finally, the relationship between the anterograde deficits in fear conditioning reported here and the retrograde deficits in that conditioning reported previously (McNally \& Westbrook, 2003) is worthy of comment. We have noted that contemporary accounts invoke the same explanation for retrograde deficits in Pavlovian fear conditioning observed following hippocampal damage, social isolation, injection of morphine, and so forth as for the anterograde deficits observed in that conditioning following hippocampal damage, adrenalectomy, and so forth. This explanation emphasizes the failure of a configural or conjunctive representational system to consolidate in memory a unified representation of the conditioning context. One reason for favoring a common interpretation of anterograde and retrograde deficits in fear conditioning has been that these deficits may be specific for the context where conditioning occurred. Nonetheless, there have been failures to detect anterograde deficits in context freezing following hippocampal damage, and when detected these deficits are frequently accompanied by deficits in freezing to noncontextual stimuli. The case for anterograde deficits emerging from a failure of consolidation of a configural or conjunctive representational system is considerably weaker than for retrograde deficits. A very similar pattern was reported in the present experiments. A history of morphine injections previously shown to produce a temporally bound and context-specific retrograde impairment in Pavlovian fear conditioning (McNally \& Westbrook, 2003) also produced anterograde impairments in that conditioning. These impairments were characterized, initially at least, by deficits in contextual and CS conditioning. These deficits did not recover across a retention interval, were absent when tested immediately after conditioning, and were determined by the presence versus absence of the CS. Thus, taken together, these findings with prolonged exposure to morphine suggest that distinct mechanisms mediate the consequences of postconditioning versus preconditioning exposures to morphine: The former appear to involve disruption in consolidation of a contextual learning, whereas the latter appear to emerge from the increased susceptibility of contextual cues to overshadowing by the discrete CS.

\section{References}

Anagnostaras, S. G., Maren, S., \& Fanselow, M. S. (1999). Temporally graded retrograde amnesia of contextual fear after hippocampal damage in rats: Within-subjects examination. Journal of Neuroscience, 19, $1106-1114$.

Bevins, R. A., McPhee, J. E., Rauhut, A. S., \& Ayres, J. J. (1997). Converging evidence for one-trial context fear conditioning with an immediate shock: Importance of shock potency. Journal of Experimental Psychology: Animal Behavior Processes, 23, 312-324.

Bourtchuladze, R., Frenguelli, B., Blendy, J., Cioffi, D., Schutz, G., \& Silva, A. J. (1994). Deficient long-term memory in mice with a targeted mutation of the cAMP-responsive element-binding protein. Cell, 79, $59-68$.

Brush, F. R. (1971). Retention of aversively motivated behavior. In F. R. Brush (Ed.), Aversive conditioning and learning (pp. 402-461). New York: Academic Press.

Fanselow, M. S. (1980). Conditional and unconditional components of post-footshock freezing in rats. Pavlovian Journal of Biological Science, $15,177-182$

Fanselow, M. S. (1986). Associative vs. topographical accounts of the immediate footshock freezing deficit in rats: Implications for the response selection rules governing species specific defense reactions. Learning and Motivation, 17, 16-39.

Fanselow, M. S. (1990). Factors governing one-trial contextual conditioning. Animal Learning \& Behavior, 18, 264-270.

Fanselow, M. S. (2000). Contextual fear, gestalt memories, and the hippocampus. Behavioral Brain Research, 110, 73-81.

Fleshner, M., Pugh, C. R., Tremblay, D., \& Rudy, J. W. (1997). DHEA-S selectively impairs contextual-fear conditioning: Support for the antiglucocorticoid hypothesis. Behavioral Neuroscience, 111, 512-517.

Gewirtz, J. C., McNish, K. A., \& Davis, M. (2000). Is the hippocampus necessary for contextual fear conditioning? Behavioral Brain Research, 110, 83-95.

Hays, W. L. (1972). Statistics for the social sciences. New York: Holt, Rinehart, \& Winston.

Holt, W., \& Maren, S. (1999). Muscimol inactivation of the dorsal hippocampus impairs contextual retrieval of fear memory. Journal of Neuroscience, 19, 9054-9062.

James, J. H., \& Wagner, A. R. (1980). One-trial overshadowing: Evidence of distributive processing. Journal of Experimental Psychology: Animal Behavior Processes, 6, 188-205.

Kamin, L. J. (1957). The retention of an incompletely learned avoidance response. Journal of Comparative and Physiological Psychology, 50, 457-460.

Kaye, H., Gambini, B., \& Mackintosh, N. J. (1988). A dissociation between one-trial overshadowing and the effect of a distractor on habituation. The Quarterly Journal of Experimental Psychology, 40B, 31-47.

Kesner, R. P., \& Connor, H. S. (1972). Independence of short- and long-term memory: A neural systems analysis. Science, 176, 432-434.

Kiernan, M. J., \& Westbrook, R. F. (1993). Effects of exposure to a to-be-shocked environment upon the rat's freezing response: Evidence for facilitation, latent inhibition, and perceptual learning. Quarterly Journal of Experimental Psychology, 46B, 271-288.

Killcross, A. S., Kiernan, M. J., Dwyer, D., \& Westbrook, R. F. (1998) Effects of retention interval on latent inhibition and perceptual learning. Quarterly Journal of Experimental Psychology, 51B, 59-74.

Kim, J. J., \& Fanselow, M. S. (1992). Modality-specific retrograde amnesia of fear. Science, 256, 675-677.

Kim, J. J., Fanselow, M. S., DeCola, J. P., \& Landeira-Fernandez, J. (1992). Selective impairment of long-term but not short-term conditional fear by the $N$-methyl-D-aspartate antagonist APV. Behavioral Neuroscience, 106, 591-596.

Kim, J. J., Rison, R. A., \& Fanselow, M. S. (1993). Effects of amygdala, hippocampus, and periaqueductal gray lesions on short- and long-term contextual fear. Behavioral Neuroscience, 107, 1093-1098.

Mackintosh, N. J., \& Reese, B. (1979). One-trial overshadowing. The Quarterly Journal of Experimental Psychology, 31B, 519-526.

Maren, S. (2001). Neurobiology of Pavlovian fear conditioning. Annual Review of Neuroscience, 24, 897-931.

Maren, S., Aharonov, G., \& Fanselow, M. S. (1997). Neurotoxic lesions of the dorsal hippocampus and Pavlovian fear conditioning in rats. Behavioral Brain Research, 88, 261-274.

Maren, S., \& Fanselow, M. S. (1997). Electrolytic lesions of the fimbria/ fornix, dorsal hippocampus, or entorhinal cortex produce anterograde deficits in contextual fear conditioning in rats. Neurobiology of Learning and Memory, 67, 142-149.

McLaren, I. P. L., Kaye, H., \& Mackintosh, N. J. (1989). An associative theory of the representation of stimuli: Applications to perceptual learning and latent inhibition. In R. G. M. Morris (Ed.), Parallel distributed processing: Implications for psychology and neurobiology (pp. 102130). New York: Clarendon.

McNally, G. P., \& Westbrook, R. F. (2003). Temporally graded, contextspecific retrograde amnesia and its alleviation by context preexposure: 
Effects of postconditioning exposures to morphine in the rat. Journal of Experimental Psychology: Animal Behavior Processes, 29, 130-142.

O'Reilly, R. C., \& Rudy, J. W. (2001). Conjunctive representations in learning and memory: Principles of cortical and hippocampal function. Psychological Review, 108, 311-345.

Pearce, J. M. (1994). Similarity and discrimination: A selective review and a connectionist model. Psychological Review, 101, 587-607.

Phillips, R. G., \& LeDoux, J. E. (1992). Differential contribution of amygdala and hippocampus to cued and contextual fear conditioning. Behavioral Neuroscience, 106, 274-285.

Phillips, R. G., \& LeDoux, J. E. (1994). Lesions of the dorsal hippocampal formation interfere with background but not foreground contextual fear conditioning. Learning \& Memory, 1, 34-44.

Pugh, C. R., Fleshner, M., \& Rudy, J. W. (1997). Type II glucocorticoid receptor antagonists impair contextual but not auditory-cue fear conditioning in juvenile rats. Neurobiology of Learning and Memory, 67, 75-79.

Pugh, C. R., Kumagawa, K., Fleshner, M., Watkins, L. R., Maier, S. F., \& Rudy, J. W. (1998). Selective effects of peripheral lipopolysaccharide administration on contextual and auditory-cue fear conditioning. Brain Behavior and Immunity, 12, 212-229.

Pugh, C. R., Tremblay, D., Fleshner, M., \& Rudy, J. W. (1997). A selective role for corticosterone in contextual-fear conditioning. Behavioral Neuroscience, 111, 503-511.

Rescorla, R. A., \& Wagner, A. R. (1972). A theory of Pavlovian conditioning: Variations in the effectiveness of reinforcement and nonreinforcement. In A. H. Black \& W. F. Prokasy (Eds.), Classical conditioning: II. Current research and theory (pp. 64-99). New York: AppletonCentury-Crofts.

Rodger, R. S. (1967). Type II errors and their decision basis. British Journal of Mathematical and Statistical Psychology, 20, 187-204.

Rudy, J. W. (1996). Postconditioning isolation disrupts contextual conditioning: An experimental analysis. Behavioral Neuroscience, 110, $238-$ 246.
Rudy, J. W., Barrientos, R. M., \& O’Reilly, R. C. (2002). Hippocampal formation supports conditioning to memory of a context. Behavioral Neuroscience, 116, 530-538.

Rudy, J. W., Kuwagama, K., \& Pugh, C. R. (1999). Isolation reduces contextual but not auditory-cue fear conditioning: A role for endogenous opioids. Behavioral Neuroscience, 113, 316-323.

Rudy, J. W., \& Pugh, C. R. (1998). Time of conditioning selectively influences contextual fear conditioning: Further support for a multiplememory systems view of fear conditioning. Journal of Experimental Psychology: Animal Behavior Processes, 24, 316-324.

Sutherland, N. S., \& Mackintosh, N. J. (1971). Mechanisms of animal discrimination learning. New York: Academic Press.

Wagner, A. R. (1981). SOP: A model of automatic memory processing in animal behavior. In N. E. Spear \& R. R. Miller (Eds.), Information processing in animals: Memory mechanisms (pp. 95-128). Hillsdale, NJ: Erlbaum.

Westbrook, R. F., Good, A. J., \& Kiernan, M. J. (1997). Microinjection of morphine into the nucleus accumbens impairs contextual learning in rats. Behavioral Neuroscience, 111, 996-1013.

Westbrook, R. F., Greeley, J. D., Nabke, C. P., \& Swinbourne, A. L. (1991). Aversive conditioning in the rat: Effects of a benzodiazepine and of an opioid agonist and antagonist on conditioned hypoalgesia and fear. Journal of Experimental Psychology: Animal Behavior Processes, 17, 219-230.

Young, S. L., Bohenek, D. L., \& Fanselow, M. S. (1994). NMDA processes mediate anterograde amnesia of contextual fear conditioning induced by hippocampal damage: Immunization against amnesia by context preexposure. Behavioral Neuroscience, 108, 19-29.

Received June 17, 2002 Revision received March 17, 2003 Accepted March 19, 2003

\section{Low Publication Prices for APA Members and Affiliates}

Keeping you up-to-date. All APA Fellows, Members, Associates, and Student Affiliates receive-as part of their annual dues-subscriptions to the American Psychologist and APA Monitor. High School Teacher and International Affiliates receive subscriptions to the APA Monitor, and they may subscribe to the American Psychologist at a significantly reduced rate. In addition, all Members and Student Affiliates are eligible for savings of up to $60 \%$ (plus a journal credit) on all other APA journals, as well as significant discounts on subscriptions from cooperating societies and publishers (e.g., the American Association for Counseling and Development, Academic Press, and Human Sciences Press).

Essential resources. APA members and affiliates receive special rates for purchases of APA books, including the Publication Manual of the American Psychological Association, and on dozens of new topical books each year.

Other benefits of membership. Membership in APA also provides eligibility for competitive insurance plans, continuing education programs, reduced APA convention fees, and specialty divisions.

More information. Write to American Psychological Association, Membership Services, 750 First Street, NE, Washington, DC 20002-4242. 